\title{
PERFIL DAS VÍTIMAS INTOXICADAS POR AGROTÓXICOS NO ESTADO DO RIO GRANDE DO SUL ENTRE O PERÍODO DE 2007 A 2017
}

\section{PROFILE OF VICTIMS INTOXICATED BY PESTICIDES IN THE STATE OF RIO GRANDE DO SUL FROM 2007 TO 2017}

\author{
Vivian Tallita Pinheiro de Santana', Phelipe Magalhães Duarte ${ }^{*}$; Alysson Dias Dalmas' \\ 'UNIC Educacional - Primavera do Leste, Mato Grosso, Brasil \\ *Autor correspondente: Endereço: Avenida David Riva, n 315, apartamento 7, Bairro Jardim Riva, Primavera \\ do Leste-MT, CEP: 78850-000. Telefone: (66) 98466-5279; e-mail: duarte.phe@gmail.com;
}

\section{RESUMO}

A contaminação por agrotóxicos tem despertado atenção crescente, tendo em vista suas consequências para a saúde humana e do meio ambiente. Diante dos problemas associados ao manejo desses produtos, o presente trabalho objetivou delinear o perfil dos casos e vítimas de intoxicados por agrotóxicos, notificados junto ao SINAN, no período de 2007 a 2017, para o estado do Rio Grande do Sul - Brasil. Realizou-se um levantamento epidemiológico retrospectivo, quantitativo, descritivo, transversal e documental das ocorrências. Foram registrados 1678 casos, notificados mais abundantemente nos anos de 2017, 2016, 2015 e 2014 e nos meses de novembro, janeiro, dezembro e fevereiro. As vítimas mais comumente afetadas residiam em zona rural, com faixa etária entre 20-39 anos, escolaridade completa entre a faixa de $5^{\text {a }}$ $8^{a}$ série seguido de $1^{a}-4^{a}$ série completa, raça branca e sexo masculino. A maioria dos casos tiveram circunstâncias acidentais, seguido de uso habitual e tentativa de suicídio. A exposição ocorreu mais predominantemente em ambiente de trabalho e de forma aguda-única. Os casos tiveram a classificação final descrita mais recorrentemente como intoxicação. $O$ critério de confirmação mais ocorrente foi o clínico, seguido de clínico-epidemiológico. A maioria das vítimas acometidas evoluiu para cura sem sequelas, entretanto foram observados 41 óbitos por intoxicação. Os municípios de maior incidência foram: Bento Gonçalves, Cachoeira do Sul, Progresso, Venâncio Aires e Santa Maria. A utilização de agrotóxico é um problema sócio ambiental historicamente condicionado pelo modelo de desenvolvimento do país, sendo essencial que medidas preventivas sejam adotadas, afim de reduzir os riscos associados a esse uso e com repercussões para a saúde.

Palavras-chave: intoxicação; vigilância epidemiológica; agricultura; agrotóxicos.

\section{ABSTRACT}

Contamination by pesticides has attracted increasing attention in view of its consequences for human health and the environment. Looking at some problems associated with the management of these products, the present work is aimed to delineate the profile of the cases and victims intoxicated by pesticides, notified to SINAN, from 2007 to 2017, for the state of Rio Grande do Sul Brazil. A retrospective, quantitative, descriptive, cross-sectional and documentary epidemiological survey of the occurrences was carried out. There were 1678 cases, reported more abundantly in the years 2017, 2016, 2015 and 2014 and in the months of November, January, December and February. The most commonly affected victims lived in rural areas, ranging in age from 20-39 years old, with complete schooling between the 5th and 8th grade followed by the 1st and 4th full grade, 
white and male. Most of the cases had accidental circumstances, followed by habitual use and attempted suicide. The exposure occurred most predominantly in the work environment and in an acute-only form. The cases had the final classification described more frequently as intoxication. The most frequent confirmatory criterion was the clinical, followed by clinical-epidemiological. The majority of affected patients were cured and stayed without sequels, however 41 deaths due to intoxication were observed. The cities with the highest incidence were: Bento Gonçalves, Cachoeira do Sul, Progresso, Venâncio Aires and Santa Maria. The use of pesticides is a socioenvironmental problem historically conditioned by the country's development model, so it is essential that preventive measures be adopted in order to reduce the risks associated with this use and with health repercussions.

Keywords: intoxication; epidemiological surveillance; agriculture; pesticides.

\section{INTRODUÇÃO}

A produção de alimentos necessária para suprir a demanda da população em crescimento constitui um desafio para esse setor. O aumento da produtividade entra em conflito com questões ambientais pois, para a intensificação desta atividade econômica, a exploração dos recursos naturais se torna cada vez mais intensa (MARCONDES; MARCONDES, 1996; PIGNATI et al., 2017; SILVA et al., 2005). Além disso, a produção de alimentos em quantidades suficiente, muitas vezes necessita estar aliada ao uso de compostos agroquímicos, incluindo fertilizantes e agrotóxicos (PIGNATI et al., 2017; SANTANA et al., 2016; SILVA; FRANCISCONI, 2004). Tais substâncias são usadas para garantir a imediata produtividade desse setor (MARCONDES; MARCONDES, 1996; RIGOTTO; VASCONCELOS; ROCHA, 2014).

No Brasil, a Lei Federal no 7.802, em seu Artigo 2, Inciso I, define os agrotóxicos e afins como os produtos e os componentes de processos físicos, químicos ou biológicos destinados ao uso no setor de produção, armazenamento e beneficiamento de produtos agrícolas, nas pastagens, na proteção de florestas nativas ou implantadas e de outros ecossistemas e também em ambientes urbano, hídricos e industriais, cuja finalidade seja alterar a composição da flora e da fauna, a fim de preservá-la da ação danosa de seres vivos considerados nocivos, bem como substâncias e produtos empregados como desfolhantes, dessecantes, estimuladores e inibidores do crescimento (BRASIL, 1989).

Já o termo pesticida é atribuído aos agentes químicos aplicados com finalidade de prevenir a ação ou destruir direta ou indiretamente agentes nocivos à lavoura, tais como: insetos, ácaros, roedores, ervas daninhas, bactérias e plantas daninhas (H.; LARA; C., 1992). E o termo defensivo agrícola tenta suavizar a utilização do termo agrotóxico, sugerindo que as plantas são completamente suscetíveis às pragas e doenças, entretanto este não deixa explícito os potenciais efeitos adversos do uso dessas substancias aos seres humanos e ao meio ambiente (INFORMATIVO CRQ III, 1997), o que veio a ser corrigido após a promulgação da Lei № 7.802/89, considerando indevido o uso do termo "defensivo agrícola" adotando, portanto, o termo "agrotóxico" como definitivo (MACHADO, 2008).

Nos países em desenvolvimento observa-se o aumento da utilização de agrotóxicos, devido à maior flexibilidade da legislação e regulamentação do uso nesses países (REBELO et al., 2011), que justifica-se pela demanda produtiva (MOTA et al., 2012). No Brasil a alta produtividade agrícola se 
deve ao grande uso de insumos químicos (CARVALHO; PIVOTO, 2011; SANTANA; MOURA; NOGUEIRA, 2013; SOARES; PORTO, 2012). O uso desses agrotóxicos envolve grande parte da produção de alimentos para o consumo interno e de exportação, sendo responsável pelas principais mercadorias distribuídas para várias partes do mundo, constituindo-se assim um dos maiores mercados mundial (AUGUSTO, L. G. S.; CARNEIRO, F. F.; PIGNATI, W.; RIGOTTO, R. M.; FRIEDRICH, K.; FARIA; BÚRIGO; FREITAS, V.M.T.; GUIDUCCI FILHO, 2012; RIGOTTO; VASCONCELOS; ROCHA, 2014).

Mesmo com os benefícios da aplicação de agroquímicos para a produtividade, a utilização desenfreada de agrotóxicos pode ocasionar um alto custo para o país devido aos possíveis danos ambientais (LUCHINI; ANDRÉA, 2000; SOARES; PORTO, 2012). O uso indiscriminado pode acarretar inúmeros problemas para o meio ambiente, contaminando o ar, a água, o solo causando a morte de animais e plantas, pois estas substâncias podem deslocar-se no ambiente através dos ventos e da água da chuva para locais distantes de onde foram utilizadas e, mesmo as regiões longínquas das áreas agricultáveis, podem ser afetadas (CHAIM; FRIGETTO; VALARINI, 1999; LARA; BARRETO, 1972; LUNA; SALES; SILVA, 2004).

Para o Brasil a fiscalização de quais produtos e os locais em que são usados trata-se de um fator desafiador devido as dimensões continentais, sendo este um dos principais consumidores mundiais de agrotóxicos (LOPES; ALBUQUERQUE, 2018; MALASPINA; ZINILISE; BUENO, 2011; SANTANA; MOURA; NOGUEIRA, 2013).

O uso indiscriminado de agrotóxicos também pode acarretar problemas de saúde para população exposta, com inúmeros casos de intoxicações por esses produtos em todo o país (SANTANA; MOURA; NOGUEIRA, 2013; SOARES; PORTO, 2012). Em geral, os trabalhadores envolvidos no uso desses agentes químicos não são adequadamente treinados para os cuidados de manejo desses produtos, o que gera preocupação a nível de Saúde Coletiva, por expor diretamente e indiretamente a população, contaminando não somente os trabalhadores que manipulam esses produtos, mas o meio ambiente e os alimentos (MOREIRA et al., 2010, 2002; PIGNATI; MACHADO, 2011; SANTANA; MOURA; NOGUEIRA, 2013).

O consumo de pesticidas tem crescido rapidamente nos países em desenvolvimento e em países emergentes (PIMENTEL, 1991), e na maioria dos casos não há controle eficaz sobre a venda e uso destes produtos (FARIA, 2012; FORGET, 1989; LOPES; ALBUQUERQUE, 2018), os equipamentos de proteção não são usados rotineiramente, não há monitoramento da exposição ocupacional e o diagnóstico e tratamento dos casos de intoxicação são falhos. Assim, intoxicações por agrotóxicos vem despertando atenção crescente, principalmente devido aos danos à saúde humana e o risco de degradação do meio ambiente, causados por seu uso crescente e, às vezes, inadequado (CARVALHO; PIVOTO, 2011; PASCHOAL, 1979).

Considerando que a utilização de agrotóxico é um problema sócio ambiental historicamente condicionado pelo modelo de desenvolvimento do país, com sérias repercussões para a área da saúde (GURGEL, 1998; JOBIM et al., 2010), faz-se necessário conhecer o perfil dos casos de intoxicação registrados nas diferentes regiões do pais, dentre estas o estado do Rio Grande do Sul, o qual possui significativa representatividade e produtividade no setor agropecuário, afim de auxiliar na elaboração de ações preventivas e reduzir os riscos associados a esse uso e com repercussões para a saúde e o meio ambiente. 


\section{MATERIAL E MÉTODOS}

O presente estudo trata-se de um estudo epidemiológico, observacional, descritivo e retrospectivo das ocorrências de intoxicações agrotóxicos no estado do Rio Grande do Sul - Brasil, entre os anos de 2007 a 2017. Para tanto foi utilizado o banco de dados de registro das informações contidas na ficha de notificação do DATASUS e SINAN (Sistema de Informação de Agravos de Notificação) e disponibilizados pelo site do Ministério da Saúde. Trata-se de uma base de acesso irrestrito, gratuito e com anonimato dos pacientes, o que dispensa aprovação do presente estudo por Comitê de Ética em Pesquisa (CEP), de acordo com a Resolução do Conselho Nacional de Saúde (CNS) nº 466, de 12 de dezembro de 2012.

\section{RESULTADOS E DISCUSSÃO}

Os dados avaliados através do presente estudo, permitiram constatar o registro de 1678 casos de intoxicação por agrotóxicos no estado do Rio Grande do Sul, ao longo do período avaliado (Tabela 1). Os riscos e perigos de intoxicações por agrotóxicos no Brasil tem se elevado com a expansão do plantio de monoculturas em algumas regiões como Sul, Sudeste e Centro-Oeste (AGOSTINETTO et al., 1998; BALDERRAMA, 2000). Em 2010 os defensivos agrícolas contabilizaram US\$ 47,6 bilhões em vendas (AGROW, 2011). Essa expansão da comercialização se deu pela crescente aplicação de herbicidas e fungicidas, bem como, no aumento participativo de importantes mercados mundiais (BARROSO; WOLFF, 2012; CROPLIFE, 2010), como o Brasil em relação à América Latina (MCDOUGALL, 2011).

O aumento de área cultivada propicia a elevação do número de intoxicações por agentes químicos crescentemente utilizados como defensivos agrícolas (BARROSO; WOLFF, 2012; CONAB, 2018; PIGNATI et al., 2017; SILVA et al., 2005). O estado do Rio Grande do Sul é um dos maiores produtores e exportadores de grãos do país (BARROSO; WOLFF, 2012). A agropecuária é fundamental para a economia regional e, de acordo com o PIB Municipal, em 2013 os setores agrícola e pecuário consistiram na engrenagem principal que movimentou a economia de 147 dos municípios do estado (FUNDAÇÃO DE ECONOMIA E ESTATÍSTICA SIEGFRIED EMANUEL HEUSER, 2015).

A região Sul é responsável por, aproximadamente, 30\% do consumo desses agroquímicos no país sendo portanto, bastante comum o uso dos agrotóxicos para melhoria da produtividade nessas áreas, o que pode propiciar a intoxicação por estes agentes, que podem proporcionar riscos ao meio ambiente e à saúde humana (JOBIM et al., 2010).

O período do ano de maior ocorrência dos casos de intoxicação por agrotóxicos no estado do Rio Grande do Sul compreendeu os meses de novembro 15,25\% (n=250), janeiro 15,68\% (n=257), dezembro $15,13 \%(n=248)$ e fevereiro $11,10 \%(n=182)$. Tais resultados possivelmente estão relacionados com o período de aplicação comum desses produtos químicos nas lavouras, que de acordo com o calendário de plantio e colheita agrícola brasileiro, ocorrem nesses mesmos meses do ano (CONAB, 2018).

O ano de maior incidência das intoxicações foi em 2017 ( $n=408)$, seguido de 2016 ( $n=262), 2015$ ( $n=181)$ e 2014 ( $n=164)$ (Tabela 1). Os resultados apontam um crescente aumento no número de casos ao longo dos anos, o que pode ter sido propiciado pelo aumento da área cultivada na região pesquisada (CONAB, 2018) e, consequentemente, pelo crescimento uso de defensivos, possibilitando maior contato com esses produtos e consequentes intoxicações.

As vítimas mais comumente afetadas residiam em área rural 63,05\% (n=1058), seguido da urbana 33,61\% (N=564) (Tabela 1). Conforme o Censo Demográfico de 2010 a população residente na 
zona rural do Rio Grande do Sul, era de aproximadamente 1,6 milhões de pessoas, o que representa cerca de 15\% da população total do estado (INSTITUTO BRASILEIRO DE GEOGRAFIA E ESTATÍSTICA - IBGE, 2014), essa população comumente está mais susceptível ao contato com agrotóxicos devidos as atividades laborais desenvolvidas estarem comumente relacionadas a utilização desses produtos (DELGADO; PAUMGARTTEN, 2004; MOREIRA et al., 2002).

Quanto a escolaridade, as vítimas apresentavam formação de $5^{\mathrm{a}}-8^{\mathrm{a}}$ série em $19,67 \%$ (n=330) dos casos, seguidos de $1^{\mathrm{a}}-4^{\mathrm{a}}$ série em 14,06\% (n=236) e Ensino médio completo em 9,30\% ( $\left.\mathrm{n}=156\right)$, porém, 29,38\% (n=493) dos casos não houve declaração do grau de escolaridade, sendo registrados como Ignorado/Branco (Tabela 1). No estudo em que avaliaram os problemas de saúde do trabalhador rural, verificou-se que escolaridade média dessas vítimas foi de 4,8 anos (FARIA et al., 2000).

A baixa escolaridade apresentada pelas pessoas expostas pode ser um fator determinante para a intoxicação, pois pode dificultar a compreensão das instruções de manejo do produto. Níveis menores de escolaridade também foram verificados no Piauí através do estudo dos trabalhadores envolvidos em intoxicações com agrotóxicos (SANTANA et al., 2016). A pouca escolarização provavelmente influencia no correto entendimento das informações disponíveis na rotulagem dos produtos e no receituário agronômico, assim como, também limita o acesso às informações de segurança em geral (ARAÚJO; NOGUEIRA; AUGUSTO, 2000; MOREIRA et al., 2002; MÜLLER; FARIA, 2004).

A faixa etária da vítima mais prevalente neste estudo foi de 20-39 anos (41,95\%; n=704), seguido de 40-59 anos (36,35\%; n=610), 15-19 anos (6,26\%; n=105) e 60-64 anos (5,36\%; n=90) (Tabela 1). A partir desses resultados observa-se que a maioria dos casos envolveu a população de faixa etária economicamente ativa, assim como registrado em estudo anterior (BARROSO; WOLFF, 2009) também realizado no Rio Grande do Sul, em que evidenciaram que 47,5\% das intoxicações ocorriam na faixa etária entre 20 e 39 anos. De acordo com estudo de base populacional realizado em uma região agrícola do Rio Grande do Sul, os autores descreveram o perfil sócio demográfico e prevalência de algumas morbidades de trabalhadores rurais, e a idade média observada foi de 41,4 anos (FARIA et al., 2000).

A maior proporção de vítimas de intoxicações na faixa etária de adultos jovens também foi observado em estudos em que avaliaram o perfil epidemiológico das intoxicações por agrotóxicos no Brasil (MALASPINA; ZINILISE; BUENO, 2011), o perfil de intoxicados por Aldicarb registrados no Instituto Médico Legal no Estado do Rio de Janeiro durante o período de 1998 a 2005 (CRUZ et al., 2013) e no estudo das intoxicações no estado de Mato Grosso (OLIVEIRA; SUCHARA, 2014).

A raça das vítimas de maior prevalência foi a branca $84,80 \%(n=1423)$, seguida de parda $6,85 \%$ $(n=115)$ e preta $3,22 \%(n=54)$. As prevalências observadas para cada raça pode ser justificada pelas diferentes proporções destas na população do estado, pois de acordo com o censo realizado pelo IBGE em 2010, uma maior proporção da população na região estão alocada na raça branca, representando cerca de $83 \%$, a raça parda representou 10,5\%, seguido da preta com 5,5\% (INSTITUTO BRASILEIRO DE GEOGRAFIA E ESTATÍSTICA, 2010).

Apenas três vítimas estavam gestantes, das quais duas $(0,12 \%)$ no $2^{\circ}$ trimestre gestacional e uma $(0,06 \%)$ no $3^{\circ}$ trimestre. No entanto, a maioria das ocorrências registradas para o presente estudo envolveu vítimas do sexo masculino (75,51\%; n=1267). A maior proporção de casos envolvendo vítimas do gênero masculino também foi observado em diferentes estudos de intoxicações (CRUZ et al., 2013; MOREIRA et al., 2010; PRESGRAVE; CAMACHO; VILLAS BOAS, 2009; RAMOS; COLLI; SANCHES, 2017). 
A predominância de intoxicações em vítimas do sexo masculino também foi registrada em outros estudos em que foram avaliados os casos com trabalhadores rurais. Estes profissionais estão frequentemente em contato com produtos químicos, o que podem causar intoxicação se usados inadequadamente (RANGEL, CÁSSIA DE FÁTIMA; ROSA, ANA CRISTINA SIMÕES; SARCINELLI, 2011; REBELO et al., 2011; SCHMIDT; GODINHO, 2006).

A maioria das intoxicações apresentaram circunstancias acidentais (40,35\%; $n=677)$, seguido de uso habitual $(23,48 ; n=394)$, tentativa de suicídio (16,92\%; n=284) e ambiental (11,74\%; n=197). As circunstâncias de intoxicação acidentais e uso habitual somadas $(63,83 \%)$ possivelmente estão relacionadas entre si com à atividade laboral desenvolvida pelas vítimas, que no presente estudo corresponderam à $64,78 \%$ (n=1087) dos casos. No estudo sobre intoxicações por medicamentos e agrotóxicos no Brasil de 1999 a 2002, os autores também registraram casos acidentais de intoxicações por agrotóxicos dentre os mais frequentes (MENDONÇA; MARINHO, 2005).

Na avaliação da mortalidade por acidentes de trabalho entre trabalhadores da agropecuária no Brasil entre 2000-2010, os autores constataram a ocorrência de riscos de acidentes relacionados ao uso de agrotóxicos (FERREIRA-DE-SOUSA; SANTANA, 2016). Conforme proposto por Wesseling et al. (1993) e Mehler et al. (2006) as intoxicações por agrotóxicos ocorrem prevalentemente em trabalhadores ligados a atividade agropecuária, e dentre os fatores condicionantes para tais intoxicações está a falta ou utilização inadequada de equipamentos de proteção individual (MEHLER LN, SCHENKER MB, ROMANO PS, 2006; WESSELING; CASTILLO; ELINDER, 1993).

Ao avaliar os fatores de risco associados ao regime de uso de agrotóxicos em Minas Gerais e, os autores apontaram maior gravidade dos casos de intoxicação dos trabalhadores que não utilizaram de EPIs (SOARES; ALMEIDA; MORO, 2003). Para evitar a exposição e assegurar a proteção à saúde o trabalhador necessita desenvolver a percepção do risco e adotar regras básicas de segurança (BARROSO; WOLFF, 2012)

Os casos em que as circunstâncias da intoxicação se deram por uso habitual podem também ter relação com a deficiência quanto ao conhecimento técnico para manipulação e aplicação desses agentes químicos e não utilização de equipamentos de proteção pelos aplicadores (DELGADO; PAUMGARTTEN, 2004; MOREIRA et al., 2002).

No presente estudo, as tentativas de suicídios por uso de agrotóxicos representaram a terceira maior proporção das circunstâncias dos casos registrados para o Rio Grande do Sul durante o período avaliado. Vários são os fatores relacionados à tentativa de suicídio, tais como, stress, desemprego, renda, problemas no trabalho, dentre outros (LIMA et al., 2008). Além destes, a depressão e a ansiedade incluem-se nos transtornos psíquicos (ARAÚJO et al., 2007; MEYER; RESENDE; ABREU, 2007).

Os agrotóxicos estão entre os principais agentes utilizados para autoextermínio (BOTEGA; MAURO; CAIS, 2004; LOVISI et al., 2006; MARÍN-LEÓN; BARROS, 2003), e os trabalhadores rurais expostos a estes agente têm maior possibilidade de morrer por suicídio, devido a maior facilidade de contato (KRAWCZYK et al., 2014)

Na avaliação da incidência de suicídios e uso de agrotóxicos por trabalhadores rurais em Luz (MG), os autores constataram que em 57,9\% dos casos o envenenamento se deu pelo uso de agrotóxicos (MEYER; RESENDE; ABREU, 2007). Na cidade de Venâncio Aires-RS, os índices de suicídios apresentaram-se elevados e com maior prevalência na área rural (FALK, 1996). 
A exposição aos agrotóxicos em ambiente de trabalho foi registrada em $64,78 \%$ (n=1087) dos casos. Maiores proporções de intoxicações por agrotóxicos em circunstâncias ocupacionais são também foram relatadas na literatura (MALASPINA; ZINILISE; BUENO, 2011; MENDONÇA; MARINHO, 2005). Dentre os trabalhadores rurais do Rio Grande do Sul, apenas uma fração é ocupada por celetistas, em que, de acordo com dados do Cadastro Geral de Empregados e Desempregados (CAGED), em dezembro de 2015 haviam mais de 84.000 carteiras assinadas alocados neste segmento. Ainda que a maioria dos trabalhadores não possuam vínculo formal, os trabalhadores não assalariados também representam um importante papel para o segmento agropecuário (MINISTÉRIO DO TRABALHO E EMPREGO - MTE, 2016) e estes podem ainda estar mais susceptíveis as intoxicações devido a possibilidade de não receberem treinamentos adequados ou não se atentarem para a correta utilização dos EPIs.

Conforme dados da Organização Internacional do Trabalho, em torno de 70.000 intoxicações agudas e crônicas fatais por agrotóxico vitimam trabalhadores anualmente (ILO/WHO, 2005) ressaltando-se a importância desse agravo como acidente de trabalho e como problema de saúde pública (KONRADSEN, 2007).

No Rio Grande do Sul a agricultura familiar é uma das principais atividades econômicas desenvolvida pela população, e muitas vezes, a única fonte de renda. Na agricultura familiar da Serra Gaúcha, a grande maioria das propriedades rurais utilizam agrotóxicos e 75\% dos trabalhadores na agricultura estão expostos a estes produtos (FARIA et al., 2000). Esse grupo de trabalhadores é mais vulneráveis e suscetíveis a intoxicações por agrotóxicos, principalmente pela baixa escolaridade, o que pode dificultar o correto manejo desses produtos (BARROSO; WOLFF, 2012; FARIA et al., 2000).

Os tipos de exposição predominantes foram o agudo-único 75,27\% (n=1263), e agudo-repetido $15,02 \%$ ( $n=252)$. Os riscos de efeitos a saúde relacionados ao uso de agrotóxicos estão condicionados ao perfil toxicológico do produto, ao tipo e à intensidade da exposição e à susceptibilidade da população exposta (DELGADO; PAUMGARTTEN, 2004). Neste estudo, os tipos de exposição mais frequente podem ter relação com as circunstâncias acidentais, uso habitual e tentativas de suicídio que foram as mais frequentes. Tais circunstâncias comumente resultam nas intoxicações agudas, estas caracterizam-se pelas exposições únicas ou sucessivas das vítimas, em um período de 24 horas em média, promovendo sinais e sintomas súbitos na vítima ou população após contato com o agente químico (BRASIL, 2016). Essas intoxicações podem ocorrer pelo contato direto ou indireto dos manipuladores com os agentes tóxicos, além da contaminação de alimentos (TRAPÉ, 1993).

A classificação final dos casos em 53,64\% (n=900) das ocorrências foi de intoxicação e 34,51\% ( $\mathrm{n}=579)$ apenas como exposição. Para tanto, o critério de confirmação mais ocorrente foi o clínico em 52,80\% ( $n=886)$ dos casos, seguido de clínico-epidemiológico, em 31,88\% ( $\mathrm{n}=535)$. Conforme dados registrados junto ao SINITOX (Sistema Nacional de Informações Tóxico-Farmacológicas), os agrotóxicos são apontados como os responsáveis por 7,0\% das intoxicações e 37,0\% dos óbitos por este agravo (FIOCRUZ, 2000). A subnotificação é um dos problemas encontrados para estabelecer a epidemiologia dos casos, consequentemente o número de óbitos real pode ser maior (EDDLESTON et al., 2002; KONRADSEN, 2007).

A maioria das vítimas acometidas evoluiu para cura sem sequelas em 78,72\% (n=1321), entretanto foram observados óbitos por intoxicação em 2,44\% (n=41) dos casos. Resultados semelhantes referentes ao número de casos confirmados (MALASPINA; ZINILISE; BUENO, 2011) e de maioria de cura sem sequelas também foram observados nos estudos de intoxicações exógenas no município 
de Sobral-CE (SILVA FILHO, 2009) assim como na avaliação do perfil epidemiológico dos casos confirmados no Maranhão (SILVA et al., 2010), o que pode ser devido a disponibilidade de recursos de hospitalares adequados para o atendimento desses pacientes (RAMOS; COLLI; SANCHES, 2017).

Os municípios em que foram registradas as maiores incidências de intoxicação por agrotóxicos foram Bento Gonçalves 11,30\% ( $n=189$ ), Cachoeira do Sul 4,84\% ( $n=81)$, Progresso 4,78\% ( $n=80)$, Venâncio Aires 3,35\% ( $n=56)$ e Santa Maria 2,51\% ( $n=42)$, que juntos totalizam 26,79\%. Ao avaliar os casos de intoxicados por agrotóxicos de trabalhadores rurais da fruticultura na cidade de Bento Gonçalves em 2009, os pesquisadores encontraram que 4\% dos trabalhadores relataram intoxicações por agrotóxicos nos 12 meses anteriores à pesquisa e 19\% em algum momento da vida (FARIA; ROSA; FACCHINI, 2009). Notificações de intoxicação por agrotóxicos em Cachoeira do Sul são relatadas onde regionalmente predomina o cultivo de soja (BELLEI, 2017). A proximidade da cidade com o campo e o crescimento urbano na cidade de Santa Maria é apontado como um fator que pode contribuir com a ocorrência de casos de intoxicação nesse município (COSTA BEBER, 1998).

Tabela 1 - Número de casos de intoxicação por agrotóxicos registados no estado do Rio Grande do Sul, no período de 2004 a 2017, em relação aos meses do ano, zona de residência, faixa etária, grau de escolaridade, raça e sexo das vítimas, circunstância da intoxicação, relação com atividade laboral e os municípios de maior registro.

\begin{tabular}{|c|c|c|c|c|c|c|c|c|c|c|c|c|c|c|}
\hline & ণ্ঠ & ఠิ & $\stackrel{\infty}{\circ}$ & ণे & 음 & $\overline{\bar{N}}$ & $\frac{N}{\circ}$ & $\frac{m}{\circ}$ & $\frac{⿱ 亠}{\circ}$ & $\stackrel{n}{\stackrel{\circ}{\circ}}$ & $\frac{\circ}{\circ}$ & $\overline{\bar{N}}$ & $\underset{\vdash}{\stackrel{\leftarrow}{\wp}}$ & $\Delta 0$ \\
\hline $\begin{array}{l}\text { Variáveis } \\
\text { MÊS }\end{array}$ & $\mathrm{N}$ & $\mathrm{N}$ & $\mathrm{N}$ & $\mathrm{N}$ & $\mathrm{N}$ & $\mathrm{N}$ & $\mathrm{N}$ & $\mathrm{N}$ & $\mathrm{N}$ & $\mathrm{N}$ & $\mathrm{N}$ & $\mathrm{N}$ & $\mathrm{N}$ & \\
\hline Jan & 0 & 0 & 4 & 13 & 18 & 34 & 24 & 14 & 19 & 31 & 34 & 65 & 257 & 16 \\
\hline Fev & 0 & 0 & 22 & 8 & 10 & 23 & 19 & 2 & 12 & 11 & 23 & 52 & 182 & 11 \\
\hline Mar & 0 & 0 & 1 & 8 & 7 & 6 & 8 & 5 & 12 & 16 & 13 & 35 & 112 & 6,8 \\
\hline Abr & 0 & 0 & 2 & 1 & 3 & 7 & 7 & 6 & 11 & 9 & 10 & 11 & 67 & 4,1 \\
\hline Mai & 0 & 0 & 0 & 4 & 2 & 3 & 3 & 2 & 2 & 5 & 5 & 21 & 47 & 2,9 \\
\hline Jun & 0 & 0 & 2 & 0 & 3 & 6 & 1 & 3 & 2 & 5 & 7 & 8 & 37 & 2,3 \\
\hline Jul & 0 & 1 & 1 & 0 & 3 & 8 & 4 & 5 & 6 & 5 & 7 & 6 & 47 & 2,9 \\
\hline Ago & 0 & 0 & 4 & 5 & 2 & 6 & 11 & 4 & 7 & 11 & 16 & 26 & 92 & 5,6 \\
\hline Set & 1 & 0 & 1 & 6 & 11 & 10 & 12 & 9 & 13 & 13 & 24 & 44 & 144 & 8,8 \\
\hline Out & 0 & 1 & 3 & 10 & 14 & 15 & 10 & 13 & 10 & 12 & 30 & 38 & 156 & 9,5 \\
\hline Nov & 0 & 0 & 5 & 16 & 12 & 19 & 12 & 19 & 31 & 32 & 46 & 58 & 250 & 15 \\
\hline Dez & 0 & 0 & 7 & 17 & 14 & 20 & 10 & 19 & 39 & 31 & 47 & 44 & 248 & 15 \\
\hline Total & 1 & 2 & 52 & 88 & 99 & 157 & 121 & 101 & 164 & 181 & 262 & 408 & 1639 & 100 \\
\hline
\end{tabular}

ZONA DE RESIDENCIA

\begin{tabular}{l|l|l|l|l|l|l|l|l|l|l|l|l|l|l} 
Ing/Branco & 0 & 1 & 0 & 1 & 2 & 5 & 2 & 1 & 4 & 5 & 6 & 14 & 41 & 2,4 \\
\hline Urbana & 0 & 12 & 26 & 23 & 30 & 47 & 35 & 38 & 58 & 70 & 87 & 136 & 564 & 34 \\
\hline Rural & 1 & 28 & 25 & 63 & 67 & 103 & 82 & 60 & 101 & 105 & 166 & 256 & 1058 & 63 \\
\hline Periurbana & 0 & 0 & 1 & 1 & 0 & 2 & 2 & 2 & 1 & 1 & 3 & 2 & 15 & 0,9 \\
\hline Total & 1 & 41 & 52 & 88 & 99 & 157 & 121 & 101 & 164 & 181 & 262 & 408 & 1678 & 100 \\
\hline
\end{tabular}


continuação

\begin{tabular}{|c|c|c|c|c|c|c|c|c|c|c|c|c|c|c|}
\hline & ষ্ণ & ఠิ & ஜ̊ & 옹 & $\frac{\circ}{\circ}$ & $\overline{\bar{N}}$ & $\frac{N}{i}$ & $\frac{m}{i}$ & $\frac{⿱}{\circ}$ & $\frac{10}{i}$ & $\stackrel{\circ}{\stackrel{ }{N}}$ & $\hat{\bar{N}}$ & $\underset{⺊}{\stackrel{\leftarrow}{0}}$ & b゚ \\
\hline Variáveis & $\mathrm{N}$ & $\mathrm{N}$ & $\mathrm{N}$ & $\mathrm{N}$ & $\mathrm{N}$ & $\mathrm{N}$ & $\mathrm{N}$ & $\mathrm{N}$ & $\mathrm{N}$ & $\mathrm{N}$ & $\mathrm{N}$ & $\mathrm{N}$ & $\mathrm{N}$ & \\
\hline FAIXA ETÁRIA & & & & & & & & & & & & & & $\%$ \\
\hline$<1$ & 0 & 0 & 1 & 0 & 2 & 1 & 0 & 1 & 2 & 0 & 1 & 4 & 14 & 0,8 \\
\hline $01 / a b r$ & 0 & 1 & 2 & 1 & 1 & 1 & 1 & 1 & 1 & 4 & 4 & 1 & 18 & 1,1 \\
\hline 05/set & 0 & 1 & 2 & 0 & 0 & 0 & 3 & 0 & 2 & 0 & 1 & 2 & 11 & 0,7 \\
\hline 14/out & 0 & 0 & 1 & 2 & 2 & 2 & 2 & 2 & 3 & 2 & 4 & 5 & 25 & 1,5 \\
\hline $15-19$ & 0 & 1 & 7 & 8 & 10 & 14 & 6 & 9 & 6 & 8 & 15 & 21 & 105 & 6,3 \\
\hline $20-39$ & 1 & 17 & 27 & 26 & 45 & 54 & 54 & 45 & 74 & 84 & 104 & 173 & 704 & 42 \\
\hline $40-59$ & 0 & 18 & 11 & 37 & 34 & 76 & 43 & 32 & 52 & 62 & 89 & 155 & 610 & 36 \\
\hline $60-64$ & 0 & 0 & 1 & 6 & 2 & 4 & 7 & 6 & 10 & 14 & 17 & 23 & 90 & 5,4 \\
\hline $65-69$ & 0 & 2 & 0 & 3 & 2 & 3 & 1 & 3 & 7 & 3 & 13 & 14 & 51 & 3 \\
\hline $70-79$ & 0 & 1 & 0 & 3 & 1 & 2 & 4 & 2 & 7 & 3 & 12 & 8 & 43 & 2,6 \\
\hline $80 \mathrm{e}+$ & 0 & 0 & 0 & 2 & 0 & 0 & 0 & 0 & 0 & 1 & 2 & 2 & 7 & 0,4 \\
\hline Total & 1 & 41 & 52 & 88 & 99 & 157 & 121 & 101 & 164 & 181 & 262 & 408 & 1678 & 100 \\
\hline
\end{tabular}

\begin{tabular}{|c|c|c|c|c|c|c|c|c|c|c|c|c|c|c|}
\hline ESCOLARIDADE & & & & & & & & & & & & & & $\%$ \\
\hline Ign/Branco & 0 & 10 & 14 & 28 & 32 & 45 & 21 & 35 & 47 & 69 & 76 & 116 & 493 & 29 \\
\hline Analfabeto & 0 & 2 & 0 & 0 & 0 & 4 & 0 & 0 & 2 & 2 & 4 & 3 & 17 & 1 \\
\hline $1^{\circ}$ a $4^{\circ}$ série & 0 & 5 & 7 & 15 & 9 & 29 & 29 & 20 & 22 & 23 & 30 & 47 & 236 & 14 \\
\hline $4^{\circ}$ série completa & 0 & 8 & 6 & 5 & 7 & 15 & 10 & 3 & 19 & 19 & 16 & 37 & 146 & 8,7 \\
\hline $5^{\circ}$ a $8^{\circ}$ série & 1 & 10 & 8 & 25 & 17 & 32 & 35 & 16 & 23 & 24 & 61 & 78 & 330 & 20 \\
\hline $\begin{array}{l}\text { Ensino Fundamental } \\
\text { Completo }\end{array}$ & 0 & 3 & 8 & 8 & 9 & 11 & 9 & 5 & 9 & 7 & 19 & 36 & 124 & 7,4 \\
\hline Ensino Médio Incompleto & 0 & 1 & 1 & 3 & 11 & 9 & 7 & 11 & 11 & 14 & 13 & 25 & 106 & 6,3 \\
\hline Ensino Médio Completo & 0 & 1 & 2 & 2 & 10 & 9 & 5 & 8 & 22 & 18 & 34 & 45 & 156 & 9,3 \\
\hline $\begin{array}{l}\text { Educação Superior } \\
\text { Incompleta }\end{array}$ & 0 & 0 & 0 & 0 & 1 & 0 & 1 & 0 & 2 & 0 & 2 & 9 & 15 & 0,9 \\
\hline Educação Superior Completa & 0 & 0 & 1 & 1 & 0 & 1 & 0 & 1 & 2 & 1 & 1 & 6 & 14 & 0,8 \\
\hline Não se aplica & 0 & 1 & 5 & 1 & 3 & 2 & 4 & 2 & 5 & 4 & 6 & 6 & 41 & 2,4 \\
\hline Total & 1 & 41 & 52 & 88 & 99 & 157 & 121 & 101 & 164 & 181 & 262 & 408 & 1678 & 100 \\
\hline RAÇA & & & & & & & & & & & & & & $\%$ \\
\hline Ign/Branco & 0 & 1 & 8 & 1 & 3 & 5 & 0 & 3 & 15 & 10 & 14 & 16 & 76 & 4,5 \\
\hline Branca & 1 & 37 & 32 & 83 & 86 & 136 & 107 & 89 & 131 & 150 & 221 & 347 & 1423 & 85 \\
\hline Preta & 0 & 1 & 5 & 0 & 4 & 5 & 4 & 1 & 8 & 5 & 8 & 13 & 54 & 3,2 \\
\hline Amarela & 0 & 0 & 0 & 0 & 2 & 0 & 0 & 0 & 0 & 0 & 0 & 0 & 2 & 0,1 \\
\hline Parda & 0 & 2 & 7 & 4 & 4 & 11 & 10 & 8 & 10 & 15 & 16 & 28 & 115 & 6,9 \\
\hline Indígena & 0 & 0 & 0 & 0 & 0 & 0 & 0 & 0 & 0 & 1 & 3 & 4 & 8 & 0,5 \\
\hline Total & 1 & 41 & 52 & 88 & 99 & 157 & 121 & 101 & 164 & 181 & 262 & 408 & 1678 & 100 \\
\hline
\end{tabular}


conclusão

\begin{tabular}{|c|c|c|c|c|c|c|c|c|c|c|c|c|c|c|}
\hline & ষ্ণ & હิ) & $\stackrel{\infty}{8}$ & 옹 & $\frac{\circ}{i}$ & $\overline{\bar{N}}$ & $\frac{N}{i}$ & $\frac{m}{\grave{N}}$ & $\frac{⿱ 亠}{\circ}$ & $\frac{n}{8}$ & $\frac{\circ}{\circ}$ & $\frac{\hat{\sigma}}{\grave{N}}$ & $\underset{⺊}{\stackrel{\leftarrow}{\circ}}$ & ถீ \\
\hline Variáveis & $\mathrm{N}$ & $\mathrm{N}$ & $\mathrm{N}$ & $\mathrm{N}$ & N & $\mathrm{N}$ & $\mathrm{N}$ & $\mathrm{N}$ & $\mathrm{N}$ & $\mathrm{N}$ & $\mathrm{N}$ & N & $\mathrm{N}$ & \\
\hline SEXO & & & & & & & & & & & & & & $\%$ \\
\hline Ignorado & 0 & 0 & 0 & 0 & 0 & 1 & 0 & 0 & 0 & 0 & 0 & 0 & 1 & 0,1 \\
\hline Masculino & 0 & 36 & 40 & 60 & 79 & 114 & 94 & 73 & 116 & 143 & 201 & 308 & 1267 & 76 \\
\hline Feminino & 1 & 5 & 12 & 28 & 20 & 42 & 27 & 28 & 48 & 38 & 61 & 100 & 410 & 24 \\
\hline Total & 1 & 41 & 52 & 88 & 99 & 157 & 121 & 101 & 164 & 181 & 262 & 408 & 1678 & 100 \\
\hline CIRCUNSTÂNCIA & & & & & & & & & & & & & & $\%$ \\
\hline Ign/Branco & 0 & 3 & 0 & 0 & 2 & 2 & 0 & 2 & 3 & 4 & 8 & 10 & 34 & 2 \\
\hline Uso Habitual & 1 & 13 & 2 & 9 & 17 & 42 & 20 & 17 & 28 & 45 & 83 & 117 & 394 & 23 \\
\hline Acidental & 0 & 10 & 26 & 58 & 49 & 60 & 54 & 48 & 60 & 69 & 90 & 152 & 677 & 40 \\
\hline Ambiental & 0 & 7 & 7 & 5 & 7 & 16 & 21 & 5 & 41 & 22 & 20 & 44 & 197 & 12 \\
\hline Uso Terapêutico & 0 & 0 & 0 & 1 & 0 & 0 & 0 & 0 & 0 & 0 & 0 & 0 & 1 & 0,1 \\
\hline Erro de Administração & 0 & 1 & 1 & 2 & 2 & 1 & 0 & 2 & 1 & 1 & 3 & 9 & 23 & 1,4 \\
\hline Automedicação & 0 & 0 & 0 & 0 & 0 & 0 & 0 & 0 & 0 & 0 & 0 & 2 & 2 & 0,1 \\
\hline Abuso & 0 & 0 & 0 & 0 & 0 & 1 & 0 & 0 & 0 & 0 & 0 & 1 & 2 & 0,1 \\
\hline Ingestão de Alimento & 0 & 0 & 0 & 0 & 0 & 2 & 1 & 0 & 0 & 1 & 1 & 0 & 5 & 0,3 \\
\hline Tentativa de Suicídio & 0 & 7 & 16 & 12 & 20 & 28 & 18 & 25 & 28 & 34 & 43 & 53 & 284 & 17 \\
\hline Tentativa de Aborto & 0 & 0 & 0 & 0 & 0 & 0 & 0 & 0 & 0 & 0 & 0 & 1 & 1 & 0,1 \\
\hline Violência/Homicídio & 0 & 0 & 0 & 1 & 0 & 1 & 2 & 0 & 1 & 1 & 5 & 3 & 14 & 0,8 \\
\hline Outra & 0 & 0 & 0 & 0 & 2 & 4 & 5 & 2 & 2 & 4 & 9 & 16 & 44 & 2,6 \\
\hline Total & 1 & 41 & 52 & 88 & 99 & 157 & 121 & 101 & 164 & 181 & 262 & 408 & 1678 & 100 \\
\hline
\end{tabular}

\section{EXPOSIÇÃO TRABALHO}

\begin{tabular}{l|l|l|l|l|l|l|l|l|l|l|l|l|l|l}
\hline Ign/Branco & 0 & 2 & 1 & 2 & 2 & 6 & 4 & 2 & 6 & 8 & 8 & 11 & 52 & 3,1 \\
\hline $\operatorname{Sim}$ & 1 & 28 & 29 & 65 & 68 & 107 & 79 & 56 & 99 & 112 & 168 & 272 & 1087 & 65 \\
\hline Não & 0 & 11 & 22 & 21 & 29 & 44 & 38 & 43 & 59 & 61 & 86 & 125 & 539 & 32 \\
\hline Total & 1 & 41 & 52 & 88 & 99 & 157 & 121 & 101 & 164 & 181 & 262 & 408 & 1678 & 100 \\
\hline
\end{tabular}

\begin{tabular}{l|l|l|l|l|l|l|l|l|l|l|l|l|l|l} 
MUNICÍPIOS & 0 & 0 & 0 & 32 & 26 & 26 & 21 & 14 & 16 & 20 & 12 & 22 & 189 & 11 \\
\hline Bento Gonçalves & 0 & 9 & 9 & 7 & 4 & 10 & 2 & 5 & 9 & 8 & 8 & 10 & 81 & 4,8 \\
\hline Cachoeira do Sul & 0 & 0 & 0 & 21 & 3 & 19 & 7 & 3 & 7 & 2 & 1 & 17 & 80 & 4,8 \\
\hline Progresso & 0 & 0 & 0 & 0 & 9 & 3 & - & 2 & 1 & 6 & 17 & 18 & 56 & 3,4 \\
\hline Venâncio Aires & 0 & 3 & 4 & 5 & 5 & 4 & 2 & 4 & 7 & 1 & 5 & 2 & 42 & 2,5 \\
\hline Santa Maria & 0 & 12 & 13 & 65 & 47 & 62 & 32 & 28 & 40 & 37 & 43 & 69 & 448 & 27 \\
\hline Total & & &
\end{tabular}

\section{CONCLUSÃO}

As intoxicações por agrotóxicos são um problema de saúde pública no Brasil. Para o estado do Rio Grande do Sul, o presente trabalho apontou a ocorrência de 1678 casos de intoxicação por agrotóxicos ao longo do período avaliado, mais prevalente nos anos de 2017, 2016, 2015 e 2014 em que se verificou o crescimento da produção agrícola na região, a maioria registrados nos meses de 
novembro, janeiro, dezembro e fevereiro, coincidindo com o calendário de plantio e colheita agrícola brasileiro. As vítimas mais comumente afetadas residiam em zona rural, com faixa etária entre 20-39 anos, escolaridade $5^{\mathrm{a}}-8^{\mathrm{a}}$ série, raça branca e sexo masculino. Esse perfil caracteriza a população ativa nas áreas de plantio da região e que comumente estão mais susceptíveis ao contato com agrotóxicos devidos as atividades laborais desenvolvidas. A maioria dos casos tiveram circunstancia acidentais, seguido de uso habitual e tentativa de suicídio. A agricultura familiar é uma das principais atividades econômicas desenvolvida pela população, e esse grupo de trabalhadores é mais vulneráveis e suscetíveis a intoxicações por agrotóxicos, principalmente devido à baixa escolaridade. A maioria das vítimas acometidas evoluiu para cura sem sequelas, entretanto foram observados 41 óbitos por intoxicação. Os municípios onde houveram maior incidência: foram Bento Gonçalves, Cachoeira do Sul, Progresso, Venâncio Aires e Santa Maria. O crescimento demográfico e a aproximação da zona urbana em direção ao meio rural aumentam a possibilidade do contato de pessoas com agrotóxicos. Assim, conhecer as peculiaridades regionais do estado é imprescindível para se estabelecer medidas corretivas a fim de se reduzir os riscos do uso para os trabalhadores e com repercussões para a saúde pública e para o ambiente.

\section{REFERÊNCIAS}

AGOSTINETTO, D. et al. Utilização de equipamentos de proteção individual e intoxicações por agrotóxicos entre fumicultores do Município de Pelotas - RS. Ecotoxicologia e Meio Ambiente, v. 8 , p. 45-56, 1998.

AGROW. Mixed results for top 20 companies. Agribusiness Intelligence / Informa, n. 621, p. 2, ago. 2011.

ARAÚJO, A. C.; NOGUEIRA, D. P.; AUGUSTO, L. G. Impacto dos praguicidas na saúde: estudo da cultura de tomate. Revista de Saúde Pública, v. 34, n. 3, p. 309-313, jun. 2000.

ARAÚJO, A. J. et al. Exposição múltipla a agrotóxicos e efeitos à saúde: estudo transversal em amostra de 102 trabalhadores rurais, Nova Friburgo, RJ. Ciência \& Saúde Coletiva, v. 12, n. 1, p. 115-130, mar. 2007.

AUGUSTO, L. G. S.; CARNEIRO, F. F.; PIGNATI, W.; RIGOTTO, R. M.; FRIEDRICH, K.; FARIA, N. M. X.; BÚRIGO, A. C. .; FREITAS, V.M.T.; GUIDUCCI FILHO, E. Dossiê ABRASCO - Um alerta sobre os impactos dos Agrotóxicos na Saúde. Parte 2: Agrotóxicos, saúde, ambiente e sustentabilidade. Rio de Janeiro: Associação Brasileira de Saúde Coletiva DOSSIÊ, 2012.

BALDERRAMA, E. S. R. Intoxicação profissional rural por agrotóxicos no Estado do Paraná na última década. [s.l.] Universidade Federal do Paraná, 2000.

BARROSO, L. B.; WOLFF, D. B. Riscos e segurança do aplicador de agrotóxicos no Rio Grande do Sul. Disc. Scientia. Série: Ciências Naturais e Tecnológicas, v. 10, n. 1, p. 27-52, 2009.

BARROSO, L.; WOLFF, D. Riscos e segurança do aplicador de agrotóxicos no Brasil. Engenharia Ambiental - Espírito Santo do Pinhal, v. 9, n. 3, p. 87-102, 2012.

BELLEI, T. T. O uso de agrotóxicos e a prevalência de neoplasias no município de Vacaria/RS. [s.l.] Universidade de Caxias do Sul, 2017.

BOTEGA, N. J.; MAURO, M. L. F.; CAIS, C. F. S. Estudo multicêntrico de intervenção no comportamento suicida. In: B.G., W.; N.J., B. (Eds.). Comportamento suicída. Porto Alegre, RS: ARTMED, 2004. p. 123-40. 
BRASIL. Lei № 7.802, de 11 de julho de 1989. Disponível em: <http://www.planalto.gov.br/ccivil_03/ leis/L7802.htm\%3E. Acesso em: 12 junho. 2019.>. Acesso em: 14 de junho de 2019.

CARVALHO, N. L.; PIVOTO, T. S. Ecotoxicologia: conceitos, abrangência e importância agronômica. REMOA - UFSM, v. 2, n. 2, p. 176-192, 2011.

CHAIM, A.; FRIGETTO, R. T. S.; VALARINI, P. J. Manejo de agrotóxico e qualidade ambiental - manual técnico. 1. ed. Jaguariúna SP: EMBRAPA Meio Ambiente, 1999.

CONAB. Acompanhamento da Safra Brasileira - Grãos: safra 2017/18. Monitoramento agrícola. Brasilia: Observatório Agrícola, 2018. v. 5

COSTA BEBER, C. Santa Maria, 200 anos: História da economia do município. Santa Maria: Edição comemorativa do centenário da COSISM, 1998.

CROPLIFE. Facts and figures: The status of global agriculture. Disponível em: <\%3Cwww. improveagriculture.com>. Acesso em: 14 de junho de 2019.

CRUZ, C. DA C. et al. Perfil epidemiológico de intoxicados por Aldicarb registrados no Instituto Médico Legal no Estado do Rio de Janeiro durante o período de 1998 a 2005. Cadernos Saúde Coletiva, v. 21, n. 1, p. 63-70, mar. 2013.

DELGADO, I. F.; PAUMGARTTEN, F. J. R. Intoxicações e uso de pesticidas por agricultores do Município de Paty do Alferes, Rio de Janeiro, Brasil. Cadernos de Saúde Pública, v. 20, n. 1, p. 180-186, 2004.

EDDLESTON, M. et al. Pesticide poisoning in the developing world: a minimum pesticides list. Lancet, v. 360, n. 9340, p. 1163-7, out. 2002.

FALK, J. W. Estudo da Mortalidade no Município de Venâncio Aires - RS, no período de 1979 a 1991. Porto Alegre, RS: UFRGS, 1996.

FARIA, N. M. X. et al. Processo de produção rural e saúde na serra gaúcha: um estudo descritivo. Cadernos de Saúde Pública, v. 16, n. 1, p. 115-128, jan. 2000.

FARIA, N. M. X. Modelo de desenvolvimento, agrotóxicos e saúde: prioridades para uma agenda de pesquisa e ação. Revista Brasileira de Saúde Ocupacional, v. 37, n. 125, p. 17-50, 2012.

FARIA, N. M. X.; ROSA, J. A. R. DA; FACCHINI, L. A. Intoxicações por agrotóxicos entre trabalhadores rurais de fruticultura, Bento Gonçalves, RS. Revista de Saúde Pública, v. 43, n. 2, p. 335-344, abr. 2009.

FERREIRA-DE-SOUSA, F. N.; SANTANA, V. S. Mortalidade por acidentes de trabalho entre trabalhadores da agropecuária no Brasil, 2000-2010. Cadernos de Saúde Pública, v. 32, n. 4, p. 2000-2010, 2016.

FIOCRUZ. Sistema Nacional de Informações Tóxicofarmacológicas - SINITOX. Disponível em: <https://sinitox.icict.fiocruz.br/sites/sinitox.icict.fiocruz.br/files//tab2_brasil_2000.pdf.>. Acesso em: 14 de junho de 2019.

FORGET, G. Pesticides: necessary but dangerous poisons. International Development Research Center Report 1989. International Development Research Center Report, v. 18, p. 4-5, 1989.

FUNDAÇÃO DE ECONOMIA E ESTATístICA SIEGFRIED EMANUEL HEUSER. PIB Municipal. Portal FeE, 2015. 
GURGEL, I. D. G. Repercussão dos agrotóxicos na saúde dos agentes de saúde pública em Pernambuco. [s.l.] Centro de Pesquisa Aggeu Magalhães, Recife, 1998.

H., W.; LARA; C., B. G. Pesticidas. Química Nova, v. 15, n. 2, p. 161-166, 1992.

ILO/WHO. Joint Press Release ILO/WHO Number of Work related Accidents and Illnesses Continues to Increase ILO and WHO Join in Call for Prevention Strategies. Disponível em: <https://www.ilo. org/global/about-the-ilo/newsroom/news/WCMS_005161/lang--en/index.htm>. Acesso em: 14 de junho de 2019.

INFORMATIVO CRQ III. O dilema do uso de defensivos agrícolas. Rio de Janeiro: Conselho Regional de Química, III Região, 1997.

INSTITUTO BRASILEIRO DE GEOGRAFIA E ESTATÍSTICA - IBGE. Pesquisa Nacional por Amostra de Domicílios - PNAD. Disponível em: <https://www.ibge.gov.br/estatisticas/sociais/trabalho/9127pesquisa-nacional-por-amostra-de-domicilios.html?=\&t=o-que-e>. Acesso em: 14 de junho de 2019.

JOBIM, P. F. C. et al. Existe uma associação entre mortalidade por câncer e uso de agrotóxicos?: Uma contribuição ao debate. Ciência \& Saúde Coletiva, v. 15, n. 1, p. 277-288, jan. 2010.

KONRADSEN, F. Acute pesticide poisoning - a. Danish Medical Bulletin, v. 54, n. 1, p. 24-26, fev. 2007.

KRAWCZYK, N. et al. Suicide mortality among agricultural workers in a region with intensive tobacco farming and use of pesticides in Brazil. Journal of Occupational and Environmental Medicine, v. 56, n. 9, p. 993-1000, 2014.

LARA, W. H.; BARRETO, H. H. C. Residuos de pesticidas clorados em águas. Revista do Instituo Adolfo Lutz., v. 32, n. 1, P. 69-74, 1972.

LIMA, M. A. et al. Perfil epidemiológico das vítimas atendidas na emergência com intoxicação por agrotóxicos. Ciência, Cuidado e Saúde, v. 7, n. 3, p. 288-294, 2008.

LOPES, C. V. A.; ALBUQUERQUE, G. S. C. Agrotóxicos e seus impactos na saúde humana e ambiental: uma revisão sistemática. Saúde Debate, v. 42, n. 117, p. 518-534, 2018.

LOVISI, G. M. et al. Análise epidemiológica do suicídio no Brasil entre 1980 e 2006 Epidemiological analysis of suicide in Brazil from 1980 to 2006. Revista Brasileira de Psiquiatria, v. 31, n. Supl II, p. 86-94, 2006.

LUCHINI, L. C.; ANDRÉA, M. M. DE. Comportamento ambiental de agrotóxicos. Horticultura Brasileira, v. 18, p. 33-35, 2000.

LUNA, A. J.; SALES, L. T.; SILVA, R. F. Agrotóxicos: responsabilidade de todos. Uma abordagem da questão dentro do paradigma do desenvolvimento sustentável, 2004. Disponível em: <http://www. segurancaetrabalho.com.br/download/agrotoxicos-responsabilidade.doc.> Acesso em: 14 de junho de 2019.

MACHADO, P. A. L. Polvição por agrotóxicos. In: MACHADO, P. A. L. (Ed.). Direito ambiental brasileiro. São Paulo: Malheiros, 2008.

MALASPINA, F. G.; ZINILISE, M. L.; BUENO, P. C. Perfil epidemiológico das intoxicações por agrotóxicos no Brasil, no período de 1995 a 2010 . Cadernos de Saúde Coletiva, v. 19, n. 4, p. 425434, 2011. 
MARCONDES, M. E.; MARCONDES, H. B. Evolução e Biodiversidade: O que nós temos com isso? São Paulo: Scipione, 1996.

MARÍN-LEÓN, L.; BARROS, M. B. A. Mortes por suicídio: diferenças de gênero e nível socioeconômico. Revista de Saúde Pública, v. 37, n. 3, p. 357-363, jun. 2003.

MCDOUGALL, P. The global agrochemical market in 2010 - preliminary 275 review. Agribusiness Intelligence / Informa, 2011.

MEHLER LN, SCHENKER MB, ROMANO PS, S. S. California surveillance for pesticide-related illness and injury: coverage, bias, nd limitations. Journal Agromedicine, v. 11, n. 2, p. 67-79, 2006.

MENDONÇA, R. T.; MARINHO, J. L. Discussão sobre intoxicações por medicamentos e agrotóxicos no Brasil de 1999 a 2002. Revista Eletrônica de Farmácia, v. 2, n. 2, p. 45-63, 2005.

MEYER, T. N.; RESENDE, I. L. C.; ABREU, J. C. DE. Incidência de suicídios e uso de agrotóxicos por trabalhadores rurais em LUz (MG), Brasil. Revista Brasileira de Saúde Ocupacional, v. 32, n. 116, p. 24-30, dez. 2007.

MINISTÉRIO DO TRABALHO E EMPREGO - MTE. Cadastro Geral de Empregados e Desempregados (CAGED), Brasil, 2016.

MOREIRA, C. DA S. et al. Análise retrospectiva das intoxicações admitidas no hospital universitário da UFJF no período 2000-2004. Ciência e Saúde Coletiva, v. 15, n. 3, p. 879-888, 2010.

MOREIRA, J. C. et al. Avaliação integrada do impacto do uso de agrotóxicos sobre a saúde humana em uma comunidade agrícola de Nova Friburgo, RJ. Ciência \& Saúde Coletiva, v. 7, n. 2, p. 299-311, 2002.

MOTA, D. M. et al. Perfil da mortalidade por intoxicação com medicamentos no Brasil, 1996-2005: retrato de uma década. Ciência \& Saúde Coletiva, v. 17, n. 1, p. 61-70, 2012.

MÜLLER, N.; FARIA, X. Trabalho rural e intoxicações por agrotóxicos. Cad. Saúde Pública, v. 20, n. 5, p. 1298-1308, 2004.

OLIVEIRA, F. F. S.; SUCHARA, E. A. Perfil epidemiológico das intoxicações exógenas em crianças e adolescentes em município do Mato Grosso. Revista Paulista de Pediatria, v. 32, n. 4, p. 299-305, 2014.

PASCHOAL, A. D. Pragas, Praguicidas e a Crise Ambiental: problemas e soluções. Rio de Janeiro: Fundação Getúlio Vargas, 1979.

PIGNATI, W. A. et al. Distribuição espacial do uso de agrotóxicos no Brasil: uma ferramenta para a Vigilância em Saúde. Ciência \& Saúde Coletiva, v. 22, n. 10, p. 3281-3294, 2017.

PIGNATI, W. A.; MACHADO, J. M. H. O agronegócio e seus impactos na saúde dos trabalhadores e da população do Estado do Mato Grosso. In: MINAYO-GOMEZ, C.; MACHADO, J. M. H.; PENA, P. G. L. (Eds.). Saúde do trabalhador na sociedade brasileira contemporânea. Rio de Janeiro: Fiocruz, 2011. p. 245-272.

PIMENTEL, D. Diversification of biological control strategies in agriculture. Crop Protection, v. 10, n. 4, p. 243-253, 1 ago. 1991.

PRESGRAVE, R. DE F.; CAMACHO, L. A. B.; VILLAS BOAS, M. H. S. Análise dos dados dos Centros de Controle de Intoxicação do Rio de Janeiro, Brasil, como subsídio às ações de saúde pública. Caderno de Saúde Pública, v. 25, n. 2, p. 401-408, fev. 2009. 
RAMOS, T. O.; COLLI, V. C.; SANCHES, A. C. S. S. Indicadores epidemiológicos das intoxicações exógenas em crianças menores de 5 anos na região de Araçatuba-SP. Revinter, v. 10, n. 03, p. 86-100, out. 2017.

RANGEL, CÁSSIA DE FÁTIMA; ROSA, ANA CRISTINA SIMÕES; SARCINELLI, P. DE N. Uso de agrotóxicos e suas implicações na exposição ocupacional e contaminação ambiental. Cadernos de Saúde Coletiva, v. 19, n. 4, p. 435-42, 2011.

REBELO, F. M. et al. Intoxicação por agrotóxicos no Distrito Federal, Brasil, de 2004 a 2007 - análise da notificação ao Centro de Informação e Assistência Toxicológica. Ciência \& Saúde Coletiva Saúde Colet, v. 16, n. 8, p. 3493-3502, 2011.

RIGOTTO, R. M.; VASCONCELOS, D. P.; ROCHA, M. M. Uso de agrotóxicos no Brasil e problemas para a saúde pública. Caderno de Saúde Pública, v. 30, n. 7, p. 1-3, jul. 2014.

SANTANA, C. M. et al. Exposição ocupacional de trabalhadores rurais a agrotóxicos. Cadernos Saúde Coletiva, v. 24, n. 3, p. 301-307, 2016.

SANTANA, V. S.; MOURA, M. C. P.; NOGUEIRA, F. F. E. Mortalidade por intoxicação ocupacional relacionada a agrotóxicos, 2000-2009, Brasil. Revista de Saúde Pública, v. 47, n. 3, p. 598-606, 2013.

SCHMIDT, M. L. G.; GODINHO, P. H. Um breve estudo acerca do cotidiano do trabalho de produtores rurais: intoxicações por agrotóxicos e subnotificação. Revista Brasileira de Saúde Ocupacional, v. 31, n. 113, p. 27-40, jun. 2006.

SILVA, C. M. M. DE S.; FRANCISCONI, E. F. Agrotóxicos e ambiente. Brasília: Embrapa Informação Tecnológica, 2004.

SILVA, J. M. DA et al. Agrotóxico e trabalho: uma combinação perigosa para a saúde do trabalhador rural. Ciência \& Saúde Coletiva, v. 10, n. 4, p. 891-903, dez. 2005.

SILVA FILHO, J. DA. Intoxicações exógenas no município de sobral-ceará intoxicações exógenas no município de Sobral-CearáFortaleza.Especialização em Vigilância Sanitária Escola de Saúde Pública do Ceará, 2009.

SILVA, V. M. et al. Intoxicações exógenas notificadas no Sinan Net em 2010: Perfil Epidemiológico dos casos confirmados no Maranhão. Revista ABEN. Seção Maranhão, v. 1, n. 84, p. 84-85, 2010.

SOARES, W.; ALMEIDA, R. M. V. R.; MORO, S. Trabalho rural e fatores de risco associados ao regime de uso de agrotóxicos em Minas Gerais, Brasil. Cadernos de Saúde Pública, v. 19, n. 4, p. 1117-1127, ago. 2003.

SOARES, W. L.; PORTO, M. F. DE S. Uso de agrotóxicos e impactos econômicos sobre a saúde. Revista de Saúde Pública, v. 46, n. 2, p. 209-217, abr. 2012.

TRAPÉ, A. Z. O caso dos agrotóxicos. In: ROCHA, L. E.; RIGOTTO, R. M.; BUSCHINELLI, J. T. P. (Eds.). . Isto é Trabalho de Gente? Vida, Doença e Trabalho no Brasil. São Paulo: Vozes, 1993. p. 569-593.

WESSELING, C.; CASTILLO, L.; ELINDER, C. G. Pesticide poisonings in Costa Rica. Scandinavian Journal Work Environ Health, v. 19, n. 4, p. 227-35, 1993. 